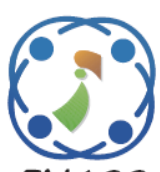

\title{
Enhancement of Data Collection Performance in Wireless Sensor Networks Based on Path-Constrained Mobile Sink
}

\author{
Mohamed Labib Borham ${ }^{1 *}$ \\ Ghada Khoriba ${ }^{2}$ \\ Mostafa-Sami Mostafa ${ }^{2}$ \\ ${ }^{1}$ Research Support Centre in Computing and Informatics (RSCCI), Computer Science Department, Faculty of \\ Computers and Artificial Intelligence, Helwan University, Egypt \\ ${ }^{2}$ Faculty of Computers and Artificial Intelligence, Helwan University, Egypt \\ * Corresponding author’s Email: mlabib@msa.eun.eg
}

\begin{abstract}
Due to the energy limitation in Wireless Sensor Networks (WSNs), most researches related to data collection in WSNs focus on how to collect the maximum amount of data from the network with minimizing the energy consumption as much as possible. Many types of research that are related to data collection are proposed to overcome this issue by using mobility with path constrained as Maximum Amount Shortest Path routing Protocol (MASP) and zone-based algorithms. Recently, Zone-based Energy-Aware Data Collection Protocol (ZEAL) and Enhanced ZEAL have been presented to reduce energy consumption and provide an acceptable data delivery rate. However, the time spent on data collection operations should be taken into account, especially concerning real-time systems, as time is the most critical factor for these systems' performance. In this paper, a routing protocol is proposed to improve the time needed for the data collection process considering less energy consumption. The presented protocol uses a novel path with a communication time-slot assignment algorithm to reduce the count of cycles that are needed for the data collection process with reduction of $50 \%$ of the number of cycles needed for other protocols. Therefore, the time and energy needed for data collection are reduced by approximately $25 \%$ and $6 \%$ respectively, which prolongs the network lifetime. The proposed protocol is called Energy-Time Aware Data Collection Protocol (ETCL).
\end{abstract}

Keywords: Data collection, Energy utilization efficiency, Routing protocols, Mobility, Path-constrained.

\section{Introduction}

With the end of 2019, Australia declared a shocking start to the fire season. The Australian forest fires, which remained for about three months between September 2019 and January 2020, are considered unprecedented natural disasters with about 19 million hectares of land and 6000 buildings burned, killing at least 40 people, and the cost of material losses is estimated to exceed 4 billion dollars. The fires had caused great losses of property and lives. The situation became very difficult until the rains contained it in February 2020.

Although Australia is one of the countries where forest fires happen frequently, the recent fire has caused great concern all over the world, especially with regards to environmental systems.

Monitoring and detection systems are the bases of

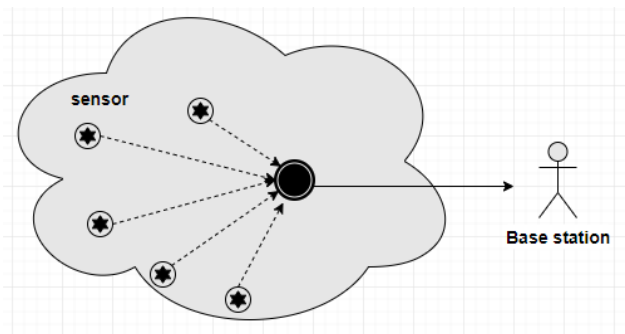

Figure. 1 Wireless sensor network

fire detection systems and play a vital role in the process. If we apply these systems effectively, we can improve the situation of fire detection on large-scale environments and many victims can be saved.

With help of Internet of Things (IoT), Wireless Sensor Networks (WSNs) play a vital role in sensing operations. WSNs are used in a large number of applications in different fields, especially in monitoring environmental applications. Its 
performance relies on sensors that collect environmental data for transmission to the base station [1].

WSNs are deployed to the target area; it is a network used to collect and process data from a specified location [2]. WSN consists of devices called sensors or nodes. Sensors are the main component forming a WSN [3]. Fig. 1. shows the wireless sensor network components.

At first sight, the rapid development of technologies help WSNs and enable them to be used in many applications, as its application is constantly increasing. WSN applications have different uses such as military applications (combat monitoring, battlefield surveillance, intruder detection), health applications (patient wearable monitoring, home assisting systems, hospital patient monitoring), environmental applications (water monitoring, air monitoring, emergency alerting). Additionally in flora and farm applications (Greenhouse Monitoring, Crop Monitoring, Livestock Farming), industrial applications (Logistics, Robotics, Machinery Health Monitoring), and urban applications (Smart Cities, Smart Homes, Transportation Systems, Structural Health Monitoring) [4]. As shown in the previous applications, WSN is used for emergency alerting, and fire detection is one of the famous systems in this area.

Data collection is one of the most important research field in WSNs, especially with the limitation of nodes' resources in CPU power, energy, memory, and bandwidth. According to these conditions, it is important to use these resources wisely and take into consideration the time needed for the data collection. Using an effective strategy in data collection and routing has a strong effect on the total time used for data gathering, and it additionally affects the sensor's energy consumption and extends the lifetime of the network. Many types of research are presented to divide the consumption of the energy between the deployed sensors.

Recently, the sink mobility trend has become the most popular strategy in WSNs to overcome the problems of the traditional methods of the static sink. [Fig. 1]. Many types of research proposed mobility in WSNs architecture were using mobile sink nodes, mobile sensing nodes, or both.

There is a relationship between the power consumption of the sensors and the range of the transmitter distance, as the power consumption of the nodes varies according to the difference in the place, and this can lead to an uneven distribution of the energy of the nodes. As is known, almost all the consumed energy of a WSN's nodes is used in transmitting messages between sensing nodes (Multi- hop approach). The target of using mobility is to minimize the total count of the messages that are transmitted through the network, as it will reduce energy consumption [5].

Nowadays, most MWSNs designs consist of static sensing nodes that sense data from the sensing area (regular sensor nodes) to direct it to the mobile sink node directly or use a multi-hop approach.

Using mobility in WSNs is required to take into consideration several features such as obtaining the location of the adjacent sensor, network topology, and the routing tables. The mobility approach has many advantages in connectivity, cost, reliability, and energy efficiency. Mobility faces many challenges such as connection discovery, perceived power management for mobility, reliable data transfer, routing protocol, and motion control $[6,7]$. The motion control is based on the trajectory type (static or dynamic) and the mobile sink speed. The trajectory of the moving sink, were static or dynamicone of the important factors in the efficiency of the data collection in MWSNs.

Through this paper, an Energy-Time Aware Data Collection Protocol (ETCL) in random topology is presented. ETCL protocol is a WSN protocol based on a path-constrained sink mobility used for data collection with the minimum time needed and takes into consideration the problem of resource limitation and energy consumption. A two-phase communication protocol is used without the need of using geographical information about the sensing area or routing table.

The presented protocol uses a novel path with a communication time-slot assignment algorithm to reduce the count of cycles that are needed for the data collection process. Therefore, the time and energy needed for data collection are reduced, which prolongs the network lifetime

Using the ns-3 simulator, ETCL protocol was implemented and performance evaluation was carried out.

The following sections introduce a brief description of WSN routing protocol that are basedon a path-constrained sink mobility used for data collection, the proposed ETCL protocol, the results of the proposed protocol and comparison with the others, finally, the paper ends with a conclusion and recommendations for future work.

\section{Related work}

As shown before, one of the important factors in data collection with mobility is motion control that depends on the type of the trajectory. Many types of researches use dissimilar trajectory of the mobile sink. 
Many types of research presented a random path where the sink node move randomly to gather the sensing nodes data using moving elements such as animals. Random- path protocols mentioned that this method is unsuited to handle the delay in data transfer, for data delivery ratio, and for data delivery efficiency. By that, almost all the researchers turned to use constrained (static) or controllable (dynamic) trajectories [8].

With sink mobility, which uses constrained or controllable trajectory, a special communication method is used that depends on the single or multihop routing protocol. Additionally, the count of the mobile sink has an important effect on the efficiency of data collection.

In path-constrained sink mobility, the mobile node is usually fixed on a moveable object that travels through a static path with constant speed, where the sensors can transfer its data directly (single hop) or by intermediate nodes (multi- hops). Although path-constrained is less complex than a controllable path, but many challenges exist, for example, the connection discovery and the connection time management through the intermediate nodes and sink node. In some scenarios, a set of proxies (intermediate nodes) has a lot of messages with a short contact time with the ME. This situation is called hotspot nodes.

S. Gao et al. [8] present a Maximum Amount Shortest Path (MASP) data collection protocol. The mentioned protocol is based on the use of a ME which moves along a constrained path (across the width or the length of the rectangle area) with multi-hop communication; the sensors that are in-range of the $\mathrm{ME}$ are selected as gateways or proxies that collect data from the network's nodes using multi-hop method. According to the node's behavior, it can be categorized into three types of nodes:

- Sink: collect data form the sub-sink nodes.

- Sub-sink: in range of sink and with a direct connection.

- Member: nodes are not in direct connection with the sink and use sub-sink nodes as intermediate nodes.

The proxies' nodes are assigned according to their length of connection time to maximize throughput with minimal energy consumption. The authors use two-phase data collection protocol (ME discovery and data gathering) to implement this scheme. MASP operates at different levels of sensor deployment.

In addition to its support of use many MEs. MASP is used to improve the data collection efficiency with a minimum amount of energy consumption; the authors introduced a scheme to overcome the problem of partial connection time used to collect data from randomly deployed sensor nodes. An integer linear programming with the help of a genetic algorithm is used to reduce the total number of hops from the sensing nodes to its destination (sub-sink node). The authors show by the implementation of this schema on the OMNET++ simulator that its performance is better than the shortest path tree (SPT) method according to the amount of data collected and power consumed.

In MASP protocol, the authors use three cycles in the discovery phase for identifying the network topology and distribute the topology information to assign the members nodes to their corresponding subsinks. As a result, the data collection time overall is consumed a large amount of energy and time. Another issue that the mentioned protocol is depends on a synchronized transmission between the mobile sink and sub-sinks nodes that are required to use a perfect synchronized clock between the nodes that are not applicable for real systems.

A. Gallegos et al. [9] introduced in detail the implementation of MASP protocol. They show that the mentioned protocol used three rounds for discovery phase only (when a mobile sink goes back and forth to the beginning of its trajectory this is called around), the first three rounds for divisions the sensed area into zones to set the routing tables, also, around for the data collection phase. Ns-3 simulator is used to implement the MASP routing protocol. The results show that the mentioned protocol is better than the ad hoc on-demand distance vector (AODV) routing protocol regarding the average energy consumption.

One of the most important factors in the data collection process is the routing protocols. Routing protocol aims to find an optimal route to reach the data to the targeted destination from its source. According to the method that is used to set the route, routing protocols are classified into three types [10, 11]:

- Proactive: routes are set in advance

- Reactive: routes are set according to source request

- Hybrid: uses both the previous types (proactive and reactive)

A. Gallegos et al. [12] presented a zone-based energy-aware data collection protocol (ZEAL) that is based on MASP. The presented protocol worked to overcome the hotspot problem by using a hybrid routing protocol. ZEAL protocol uses a homogeneous WSN (the sensors used are identical in type or capacity [13]). The presented protocol used three types of nodes as in [8] (member nodes, sub- 
Table 1. Message types in ZEAL protocol

\begin{tabular}{|c|l|}
\hline Message & \multicolumn{1}{|c|}{ Data content } \\
\hline BCST1 & assigned zone id \\
\hline UCST1 & determine sub-sink's range time \\
\hline UCST2 & $\begin{array}{l}\text { sub-sink's ideal number of nodes } \\
\text { (Mreq) within its time slot }\end{array}$ \\
\hline BCST2 & zone id, count of hops, Mreq value. \\
\hline POLL1 & sub-sink's time slot \\
\hline DATA & packets in the sub-sink queue \\
\hline POLL2 & $\begin{array}{l}\text { sleeping time, time slot time (for last } \\
\text { sub-sink in zone) }\end{array}$ \\
\hline SLP & sleeping time, zone id \\
\hline
\end{tabular}

Table 2. ZEAL setup phase

\begin{tabular}{|c|l|l|l|}
\hline $\begin{array}{c}\text { First } \\
\text { Cycle }\end{array}$ & Mobile sink & Sub-sink & $\begin{array}{c}\text { Member } \\
\text { nodes }\end{array}$ \\
\hline \multirow{4}{*}{$\begin{array}{c}\text { First- } \\
\text { half }\end{array}$} & -send BCST1 & -receive & \\
& -receive & BCST1 & \\
& BCST1 & -send & \\
& - calculate & & \\
& Mreq & & \\
Second- & - Send \\
half & UCST2 & - UCST2 & -receive \\
& & -send & BCST2 \\
& & BCST2 & -establish \\
& & & $\begin{array}{l}\text { routes to all } \\
\text { sub-sink }\end{array}$ \\
\hline
\end{tabular}

sink modes, and sink node). The mobile sink travel over a pre-defined route with constant speed multiple time. It depends on dividing the environment into independent zones according to the movement path. In this way, the mobile node gathers the sub-sink nodes' data in each zone after the sub-sink nodes queue the data from the sensors.

The journey of the mobile sink from the start point of the path to the end and return to start is considered one cycle. In ZEAL protocol many types of messages are used. Table 1, shows these messages and their content.

Processing in ZEAL protocol is done by using two phases:

\subsection{Setup phase}

This phase is done by one cycle (first and second half). The first cycle forms separate zones, the routing tables, and finally sub-sink selection for each zone. Table 2, shows messages that are sent in this phase and its order.

In the second half cycle, the mobile sink calculates the member requirements parameter (Mreq). Mreq is defined as the sub-sink's ideal number of member nodes. Mreq is calculated according to Eq. (1).

$$
\text { Mreq }=\frac{d t \times a t}{d s \times m t}-1
$$

Where:

- $d t$ : the rate of transmitting data from sub-sink to the mobile sink

- $d s$ : the rate of transmitting data from the sensing nodes to sub-sink nodes

- at: the sub-sink's time slot for sending its data to the mobile sink

- mt: mobile sink's path time

The authors used a Selective Time Assignment (SelectiveTA) algorithm with two filters to handle the assignment task of the sub-sink nodes and the mobile node. By that, the collision and overlapping communication time problems are solved.

Finally, each member node must determine its targeted sub-sink that will send its data to it. Member nodes calculate a priority for each sub-sink in its zone, and the highest priority value will be the targeted subsink. Priority value ( $p r)$ is calculated according to Eq. (2):

$$
\operatorname{Pr}=\alpha \times \text { Mreq }+\frac{1-\alpha}{\text { hopCount }}
$$

Where $\alpha$ is a weight value between 0 and 1 , and hopCount is the number of steps that are needed to reach to the sub-sink.

\subsection{Data collection phase}

After every member node determines the targeted sub-sink node in its zone, the member nodes send their data that are queued in the sub-sink. Table 3, shows the messages that are sent and its order in the data collection phase and duty cycling to reduce energy consumption by using the sleep-wake cycle.

The authors presented an implementation of the mentioned protocol using the ns-3 simulator, and the sink node travels along a rectangular area in the bottom. The results show that the ZEAL's performance is better than MASP regarding packet delivery rate and power consumption in a total of three cycles.

In ZEAL protocol, the authors select a predefined path that the mobile sink move according to a path in the bottom of the sensing area (assume the network in a rectangle shape), the selected path cause the far members nodes that are near to the upside need many hops to deliver their data to the designated subsink. The mentioned issue effects on energy consumption. Additionally, although the process of assigning the members nodes to their corresponding 
Table 3. ZEAL data collection phase

\begin{tabular}{|c|c|c|c|}
\hline $\begin{array}{l}\text { Second } \\
\text { cycle }\end{array}$ & $\begin{array}{c}\text { Mobile } \\
\text { sink }\end{array}$ & Sub-sink & $\begin{array}{c}\text { Member } \\
\text { nodes }\end{array}$ \\
\hline $\begin{array}{c}\text { First- } \\
\text { half }\end{array}$ & $\begin{array}{l}\text {-send } \\
\text { POLL1 } \\
\text {-receive } \\
\text { DATA } \\
\end{array}$ & $\begin{array}{l}\text {-receive } \\
\text { POLL1 } \\
\text {-send DATA }\end{array}$ & \\
\hline $\begin{array}{l}\text { In Duty } \\
\text { Cycling } \\
\text { Second- } \\
\text { half }\end{array}$ & $\begin{array}{l}\text {-send } \\
\text { POLL2 } \\
\text {-receive } \\
\text { DATA }\end{array}$ & $\begin{array}{l}\text { receive } \\
\text { POLL2 } \\
\text { send DATA } \\
\text { broadcast } \\
\text { SLP }\end{array}$ & $\begin{array}{l}\text {-receive SLP } \\
\text {-rebroadcast } \\
\text { SLP } \\
\text {-sleep mode }\end{array}$ \\
\hline
\end{tabular}

sub-sinks is done through one cycle. The authors use two cycles in data collection phase to achieve an acceptable ratio of data delivery. By that, the time needed for data collection is considered the same as the time needed for data collection in the MASP protocol.

A. Allam et al. [14] present an enhanced zonebased energy-aware data collection protocol for WSNs (E-ZEAL) to increase the performance of WSN in terms of data delivery rate and average power consumption. E-ZEAL is working as a hybrid routing protocol based on the ZEAL protocol but uses a controllable path. Also, the K-mean clustering algorithm is used to discover the ideal route to mobile sink movement. E-ZEAL uses through data collection processing three phases instead of the two that are used in ZEAL. The added phase was a preprocessing phase that is used in the implementation of the K-means clustering algorithm. After that, the reaming data collection phases are identical without modification as the setup and data collection phases. Another modification is done, Eq. (2) is modified to be as Eq. (3). The priority factor is normalized by adding another factor which is the Euclidean distance (dist) from the member node and the targeted subsink.

$$
\operatorname{Pr}=\frac{\text { Merq }}{\max (\text { Merq })}+\frac{\min (\text { hopCount })}{\text { hopCount }}+\frac{\min (\text { dist })}{\text { dist }}
$$

Where hopCount is the number of steps that are needed to reach to the sub-sink, and dist is the distance from the member node to the sub-sink node.

The authors in [14] use the ns-3 simulator in the experiments and show the performance by using different paths. Fig. 2 shows the different paths of ZEAL. The results show that the E-ZEAL protocol is better than ZEAL regarding data delivery and power

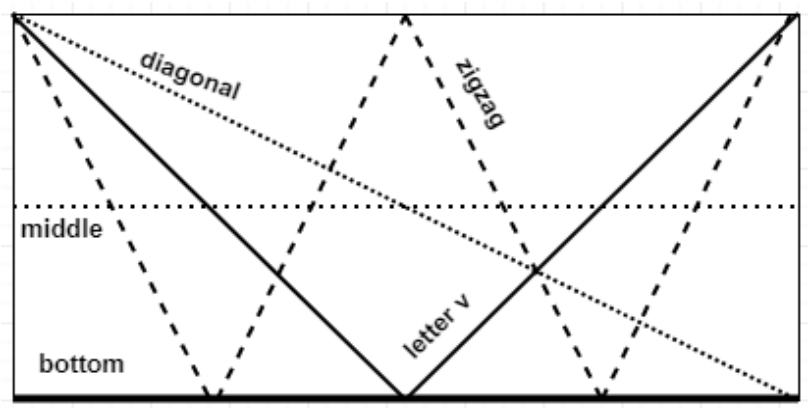

Figure. 2 ZEAL path (bottom, middle, diagonal, letter V, zigzag)

consumption.

It is noticed through the implementation of ZEAL and E-ZEAL protocol that they use three data collection cycles to collect the maximum amount of the network data. This means that more energy consumption in members nodes and delay time. ETCL, the protocol in this paper, has treated this issue and proposed a novel constrained-path to treat the mentioned issues, besides reducing the number of hops that are used to transfer the members' nodes data to its sub-sink.

Another point in E-ZEAL is that they use a controllable path, which is a type of path that may not be applicable in many WSN applications such as in a harsh environment, as it is not suitable to go through the internal of the WSN network in different positions.

\section{Energy-time aware data collection protocol (ETCL)}

In general, one of the most powerful points in using sink mobility is that the sink node can be provided by additional features such as those that can be power charged or provided by extra wireless range and data transmit rate.

Finding the ideal route proves that it has a vital effect on the routing process and consequently on data collection in WSN. Here, we use a constrained path or a controllable, finding the ideal route for the sink node is very important. The main features of the ideal route that can apply the minimum number of hop counts and reduce the distance between the nodes and their sub-sink.

Energy-Time Aware Data Collection Protocol (ETCL) is a hybrid routing protocol that is based on the ZEAL protocol. In an ETCL, a novel constrainedpath is chosen that provides a minimum number of hop counts with the minimum distance between the nodes and its sub-sink as in Fig. 3.

Assuming the environment is in a rectangle shape, $\mathrm{w}$ and 1 are the width and length of the rectangle, respectively. The perimeter of the outer rectangle (Prec) is equal: 


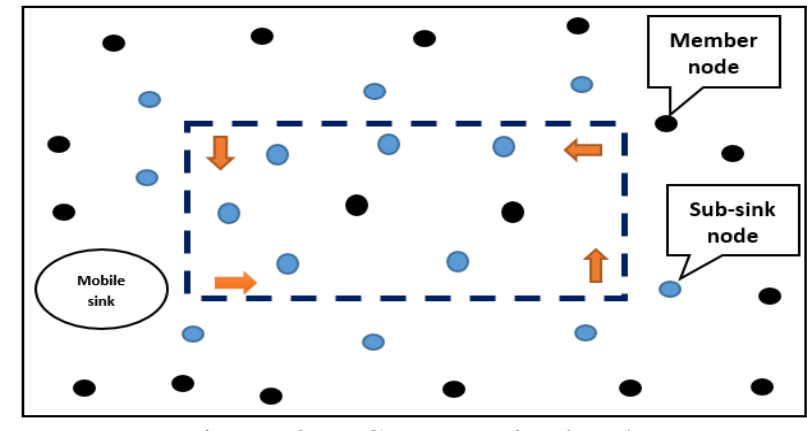

Figure. 3 ETCL constrained path

$$
P_{\text {rec }}=2 \times(w+l)
$$

From Eq. (4), the path length (Pl) for the mobile sink in the proposed protocol is equal:

$$
P l=w+l
$$

Where $\mathrm{w}$ and 1 are the width and length of the rectangle of the environment.

As mentioned before the mobile sink start to move from the start point of the path to the end and return to start is considered one cycle. With the proposed path that will be a cyclic path, it is considered that the movement from the start point to the endpoint is a half cycle. With Eq. (5), the time (t) needed to one half cycle is:

$$
t=\frac{p l}{s}
$$

Where $\mathrm{s}$ is the constant movement speed.

According to Eq. (6), the total data collection time (Ttot) is:

$$
T_{t o t}=N_{H C y} \times t
$$

Where $N_{H C y}$ is the number of half cycles

The proposed algorithm uses a novel constrainedpath that reduces the number of multi-hops that are needed to transfer the data of the member nodes to its sub-sink. Also, by using the proposed path, we can save the energy that is needed in zone partition which will not be needed in this situation. In this model, the mobile-sink goes through the internal of the WSN network and selects the sub-sink which will be near its member's nodes. In the ETCL protocol, two phases are used (setup phase and data collection phase) which are identical to the ZEAL protocol.

\section{Results and discussions}

A. Gallegos et al. [12] present the ZEAL protocol and its implementation by using the ns-3 simulator. The results show that the ZEAL protocol is better
Table 4. Different features of ZEAL, E- ZEAL, and ETCL protocols

\begin{tabular}{|l|l|l|l|}
\hline Feature & ZEAL & E-ZEAL & ETCL \\
\hline Path type & $\begin{array}{l}\text { Constraine } \\
\text { d }\end{array}$ & Controllable & Constrained \\
\hline $\begin{array}{l}\text { Method to } \\
\text { find a path }\end{array}$ & no & k-mean & no \\
\hline $\begin{array}{l}\text { Number } \\
\text { of phases }\end{array}$ & 2 & 3 & 2 \\
\hline $\begin{array}{l}\text { Method to } \\
\text { assign } \\
\text { sub-sinks }\end{array}$ & $\begin{array}{l}\text { Member } \\
\text { requiremen } \\
\text { ts number } \\
\text { of hops }\end{array}$ & $\begin{array}{l}\text { hops } \\
\text { humber of distance } \\
\text { and } \\
\text { between } \\
\text { nodes }\end{array}$ & $\begin{array}{l}\text { Member } \\
\text { requirement } \\
\text { hops }\end{array}$ \\
\hline $\begin{array}{l}\text { Zone } \\
\text { partition }\end{array}$ & $\begin{array}{l}\text { Yes } \\
\text { automatic }\end{array}$ & $\begin{array}{l}\text { Yes } \\
\text { automatic }\end{array}$ & One zone \\
\hline $\begin{array}{l}\text { Node } \\
\text { deployme } \\
\text { nt }\end{array}$ & randomly & randomly & randomly \\
\hline $\begin{array}{l}\text { Constant } \\
\text { speed }\end{array}$ & yes & yes & yes \\
\hline $\begin{array}{l}\text { Routing } \\
\text { protocol }\end{array}$ & hybrid & hybrid & hybrid \\
\hline $\begin{array}{l}\text { Data } \\
\text { transmissi } \\
\text { on }\end{array}$ & Poll & Poll & Poll \\
\hline $\begin{array}{l}\text { Energy } \\
\text { saving }\end{array}$ & yes & yes \\
\hline
\end{tabular}

than the MASP protocol in terms of delivery rate and remaining energy. In ZEAL, none of the synchronized clocks between the nodes were used, which was the main drawback in the MASP protocol. The previously mentioned protocols are based on a constrained path.

E-ZEAL protocol uses a controllable path that is defined by applying the K-mean clustering algorithm. The authors show, by implementation in the ns-3 simulator, that E-ZEAL is better than ZEAL in data delivery rate and energy consumption. Table 4, shows the different features of ZEAL, E-ZAEL, and ETCL protocols in detail.

ETCL protocol is implemented using the ns-3 simulator. Ns-3 simulator is the first known WSN simulator for discrete events network and internet systems, mainly targeted for research and learning usage. Ns-3 simulator provides a direct code execution module (DCE) that can be used with real Linux networking stack and network applications. In addition, the ns-3 simulator is marked by the ability to reuse the identical source code in testbeds of the actual applications without the need for any modification or added cost [15].

For the implementation of the proposed protocol, the network area is assumed 400m x 200m, with 120 


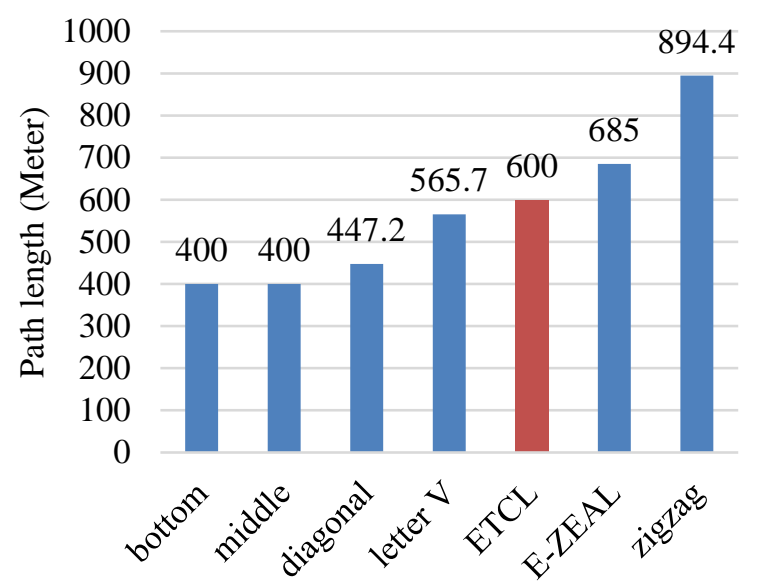

Figure. 4 Path length of ZEAL path (bottom, middle, zigzag, letter V, diagonal), E- ZEAL, and ETCL

nodes being deployed randomly. The initial energy for the nodes is set at 3000 joules with a 52-meter communication range. The mobile-sink speed is 5-20 meters/seconds. The previous assumptions are the same assumptions that are used in the implementation of the compared protocols.

ETCL protocol aims to develop the ZEAL's performance according to data collection items such as data delivery and also takes into consideration the energy consumption. The experiments are done and compared with ZEAL and E-ZEAL approaches.

Although the main target of any data collection protocol is the data delivery rate with minimum energy consumption, the time required for the data collection process is a significant factor, especially in WSN applications for emergency systems. According to Eq. (6), the path length has indirect relation with the total time required for the data collection process. Fig. 4 presents the length of the applied path in ETCL compared with different paths that are implemented in ZEAL path (bottom, middle, diagonal, letter $\mathrm{V}$, zigzag) and E-ZEAL protocols for the same network with 120 nodes.

Fig. 5 shows that the time needed for the half cycle for the different paths that are implemented in ZEAL path (bottom, middle, diagonal, letter $\mathrm{V}$, zigzag) and E-ZEAL protocols according to the movement of the mobile-sink is Eq. (6).

In the ZEAL and E-ZEAL protocols, the authors show that the data delivery is considered acceptable after two data collection cycles. This means that the experiment was done with three cycles (one cycle for setup and two cycles for data collection), which means that the mentioned protocols needed six half cycles.

The proposed protocol (ETCL) needs only three half cycles ( 1.5 cycles) to collect the data, which

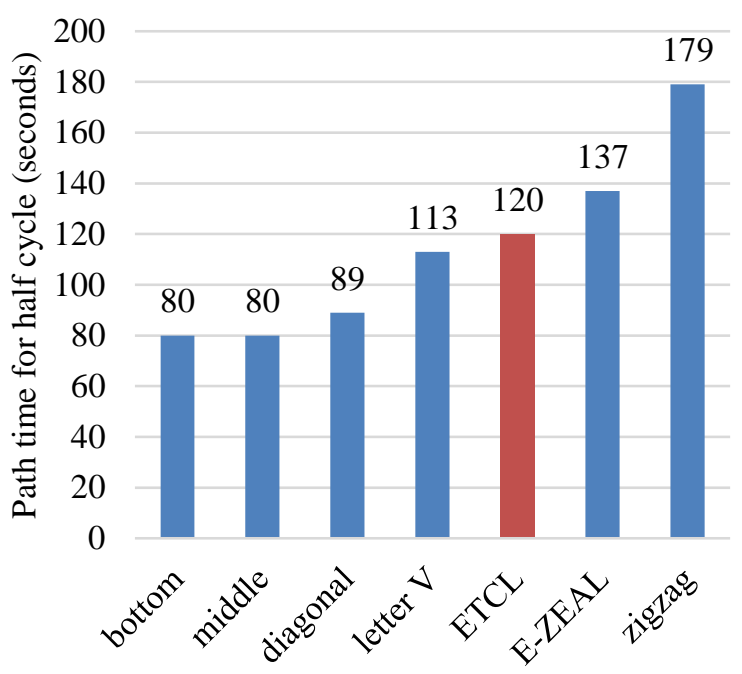

Figure. 5 Path time of ZEAL path (bottom, middle, zigzag, letter V, diagonal), E- ZEAL, and ETCL with mobile sink speed $=5$

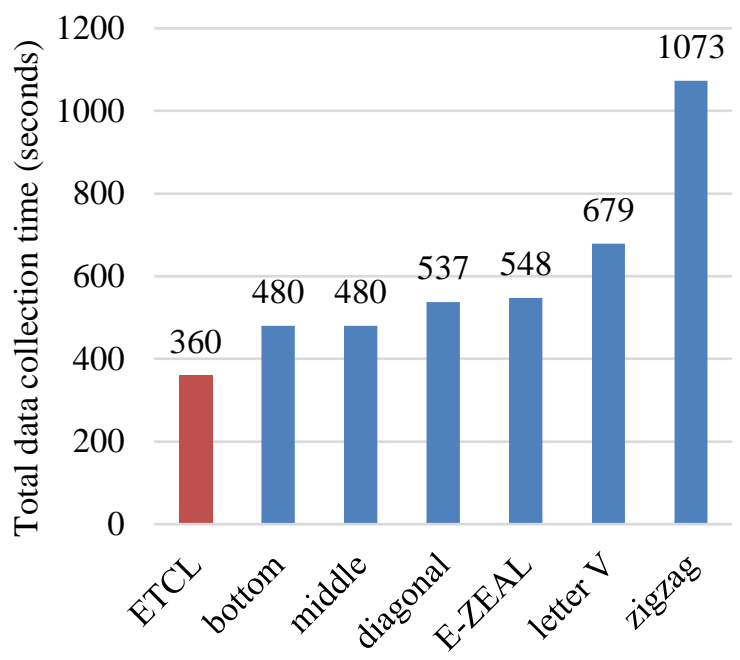

Figure. 6 Total data collection time of ZEAL path (bottom, middle, zigzag, letter V, diagonal), E- ZEAL, and ETCL with mobile sink speed $=5$

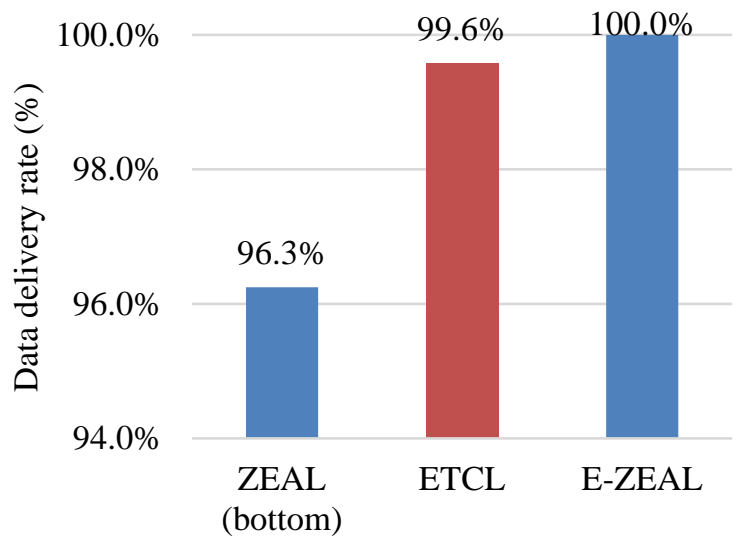

Figure. 7 Data delivery rate of ZEAL (bottom), E- ZEAL, and ETCL protocols 


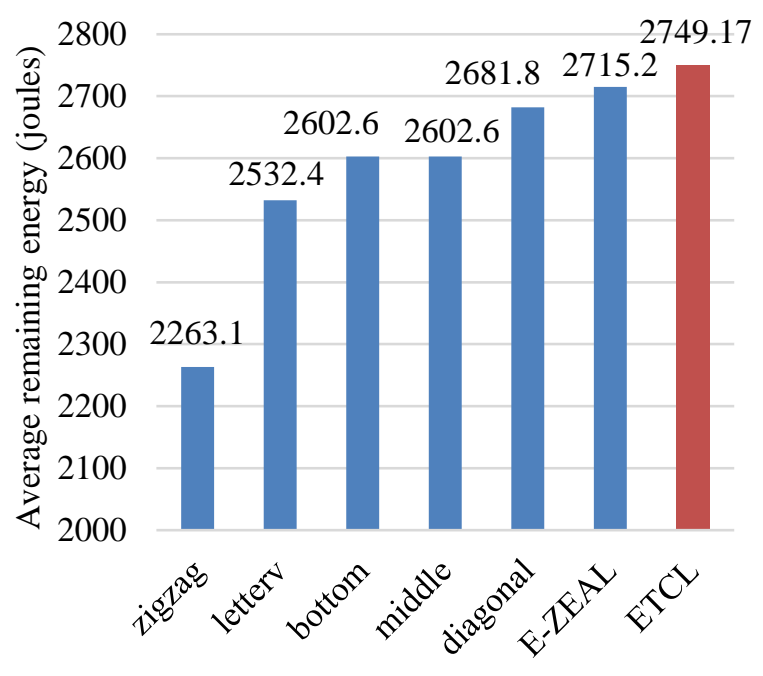

Figure. 8 Energy with ZEAL path (bottom, middle, zigzag, letter V, diagonal), E- ZEAL, and ETCL protocols

Table 5. Results of different sink-mobile speed in ETCL

\begin{tabular}{|l|l|l|l|l|}
\hline $\begin{array}{l}\text { Speed } \\
(\mathbf{m} / \mathbf{s})\end{array}$ & 5 & 10 & 15 & 20 \\
\hline $\begin{array}{l}\text { Half cycle time } \\
\text { (seconds) }\end{array}$ & 120 & 60 & 42 & 30 \\
\hline $\begin{array}{l}\text { Total } \\
\text { Data collection time } \\
\text { (seconds) }\end{array}$ & 360 & 180 & 126 & 90 \\
\hline Data Delivery/240 & 239 & 234 & 124 & 147 \\
\hline Data Delivery ratio & $100 \%$ & $98 \%$ & $52 \%$ & $61 \%$ \\
\hline $\begin{array}{l}\text { Remaining } \\
\text { Energy } \\
\text { (joules) }\end{array}$ & 2749 & 2873 & 2915 & 2935 \\
\hline $\begin{array}{l}\text { Remaining } \\
\text { energy Ratio }\end{array}$ & $92 \%$ & $96 \%$ & $97 \%$ & $98 \%$ \\
\hline
\end{tabular}

means reduction in the number of half cycles needed approximately $50 \%$ of the other protocols.

According to the path time of half cycle in Fig. 5 and Eq. (7), Fig. 6 shows that the total data collection time needed in ZEAL path (bottom, middle, diagonal, letter V, zigzag) and E-ZEAL protocols. ETCL data collection time is reduced by approximately $25 \%$, and $56 \%$ from ZEAL (bottom) and E-ZEAL, consequently.

When comparing the proposed protocol, according to the delivery rate in Fig. 7, where ZEAL and E-ZEAL use three cycles, and ETCL uses one and half cycle only, the results show that the proposed protocol has approximately the same data delivery rate as E-ZEAL protocol and better than ZEAL by $4 \%$.

Power consumption is a significant factor in the data collection protocol. The proposed protocol overcomes the protocols ZEAL and E-ZEAL in this matter. The proposed path in ETCL reduces the required cycles to collect the network data. Fig. 8 shows that the average remaining energy in ETCL is increased approximately by $6 \%$ and $1 \%$ form ZEAL (bottom) and E-ZEAL, respectively.

Sink-mobile speed affects the cycle time. The results on Table 5, show that slow speed will improve the data delivery rate but increase energy consumption and vice versa.

Although the ETCL protocol proves that data collection time is more suitable and applicable for emergency systems. The results of Figs. 9 and 10 show that the ETCL protocol provides better performance than the ZEAL (bottom path) protocol according to delivery rate and average remaining energy with a different number of nodes $(60,120$, $180)$ with network density $(6.37,12.7,19.1)$ respectively. The word "Network Density" refers to the number of nodes within the network area that has an effect on the network performance [16]. Network Density $(\mu)$ is calculated as Eq. (8):

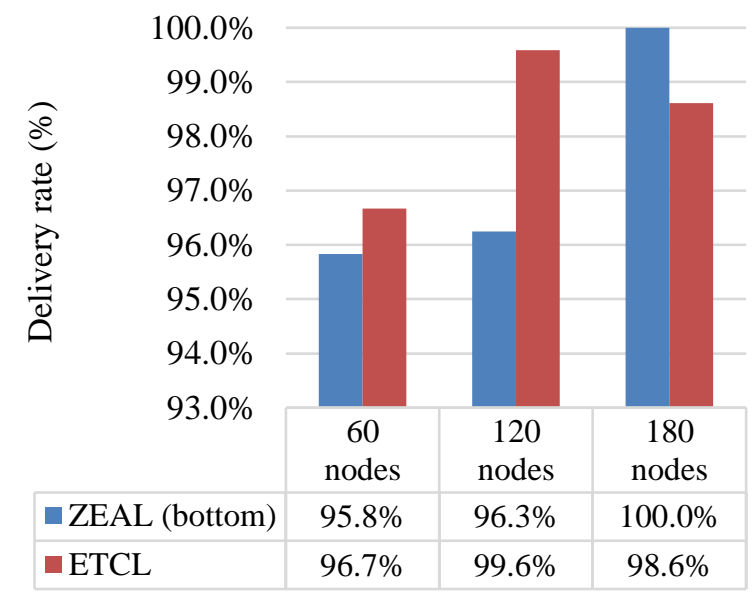

Figure. 9 Delivery rate with different number of nodes

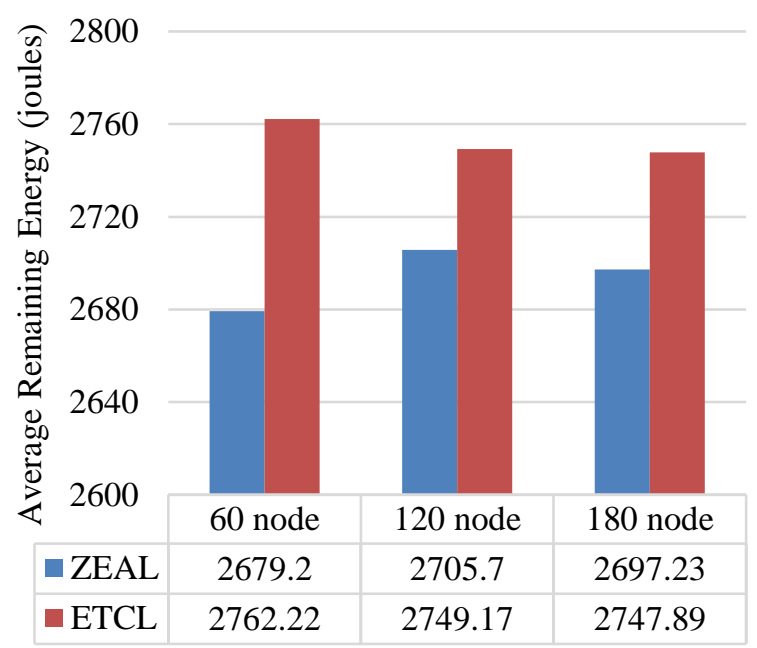

Figure. 10 Average remaining energy with different number of nodes 


$$
\mu=\frac{\left(N \pi R^{2}\right)}{A}
$$

Where $N$ is the number of nodes in area $(A)(200 \mathrm{~m}$ $\mathrm{x} 400 \mathrm{~m})$, and $R(52 \mathrm{~m})$ is the radio transmission range.

\section{Conclusion and future work}

ETCL protocol is presented to enhance the collection time needed in the data collection process, which is a very important issue for emergency systems. Although the collection time is important, we can't ignore the main objective of any data collection methods in WSN that try to overcome the power consumption and data delivery issues.

The ETCL protocol uses a novel constrained path for the sink-mobile movement to make it close to the most member nodes. The presented path shows that the process of dividing the environment into zones is not needed because the proposed path reduces the hop count between the member's nodes and its sub-sink and save energy that is used for this purpose.

Additionally, ETCL uses one and half cycle only in all the phases of data collection compared with the three cycles that are used in ZEAL and E-ZEAL protocols' results.

Ns-3 simulator is used to implement the ETCL protocol. The experiments are done with regards to the data collection time, delivery rate, and energy consumption. The performance is compared with ZEAL and E-ZAEL protocols. ETCL protocol use only three half cycles (1.5 cycles) in the data collection with reduction of $50 \%$ of the number of half cycles needed for other protocols. The results show that ETCL provides better performance in data collection time, data delivery, and power consumption. For the data collection time ETCL protocol is reduced by approximately $25 \%$, and $56 \%$ from ZEAL (bottom) and E-ZEAL, consequently. And related to the average remaining energy is increased by $6 \%$ and $1 \%$ form ZEAL (bottom) and EZEAL, respectively.

Future work can be related to enhancing the method of finding the movement path and trying to implement this protocol based on zone-based protocols.

\section{References}

[1] Akyildiz, W. Su, Y. Sankarasubramaniam, and E. Cayirci, "A survey on sensor networks", IEEE Communications Magazine, Vol. 40, No. 8, pp. $102-114,2002$.

[2] M. Keskin, "A column generation heuristic for optimal wireless sensor network design with mobile sinks", European Journal of Operational Research, Vol. 260, No. 1, pp. 291-304, 2016.

[3] T. Nguyen, K. Nguyen, and T. Trung, An Application of WSN in Smart Aquaculture Farming, Vol. 1125, Intelligent Computing in Engineering, 2020.

[4] D. Kandris, C. Nakas, D. Vomvas and G. Koulouras, "Applications of Wireless Sensor Networks: An Up-to-Date Survey", Applied System Innovation, Vol. 3, No. 1, 2020.

[5] X. Liu, and J. Wu, "A Method for Energy Balance and Data Transmission Optimal Routing in Wireless Sensor Networks", Sensors (Basel), Vol. 19, No. 13, 2019.

[6] A. Boukerche, Algorithms and Protocols for Wireless Sensor, Wiley-IEEE Press, 2008.

[7] M. Franceco, S. Das, and G. Anastasi, "Data Collection in Wireless Sensor Networks with Mobile Elements: A Survey", ACM Transactions on Sensor Networks, Vol. 8, No. 1, pp. 1-31, 2011.

[8] S. Gao, H. Zhang, and S. Das, "Efficient Data Collection in Wireless Sensor Networks With Path-constrained Mobile Sinks", IEEE Transactions on Mobile Computing, Vol. 10, No.1, pp. 592-608, 2011.

[9] A. Gallegos, T. Noguchi, T. Izumi, and Y. Nakatani, "Simulation study of Maximum Amount Shortest Path routing in Wireless Sensor Networks using Ns-3", In: Proc. of Eighth International Conf. on Ubiquitous and Future Networks (ICUFN), Vienna, Austria, pp. 198-204, 2016.

[10] R. Mukherjee, S. Roy, and A. Das, "Survey on Data Collection protocols in Wireless Sensor Networks using mobile data collectors", In: Proc. of 2nd International Conf. on Computing for Sustainable Global Development (INDIACom), New Delhi, pp. 632-636, 2015.

[11] K. Akkaya and M. Younis, "A Survey of Routing Protocols in Wireless Sensor Networks", Ad Hoc Network Journal, Vol. 3, No. 3, pp. 325-349, 2005.

[12] A. Gallegos, T. Noguchi, T. Izumi, and Y. Nakatani, "Zone-Based Energy Aware Data Collection Protocol for WSNs", IEICE Transactions on Communications, Vol. E101.B, No. 3, pp. 750-762, 2018.

[13] J. Du, H. Wang, Y. Wu, F. Jiang, and H. Huang, "A data collection approach based on mobile sinks for heterogeneous sensor networks", In: Proc. of 8th International Conf. on Wireless Communications \& Signal Processing (WCSP), Yangzhou, pp. 1-6, 2016. 
[14] A. Allam, M. Taha, and H. Zayed, "Enhanced zone-based energy aware data Collection protocol for WSNs (E-ZEAL)", Journal of King Saud University - Computers and Information sciences,

DOI: 10.1016/j.jksuci.2019.10.012.2020.

[15] https://www.nsnam.org

[16] A. Akl, T. Gayraud, and P. Berthou, "A metric for evaluating density level of wireless sensor networks", In: IFIP Wireless Days (WD), Niagara Falls, pp. 1-3, 2011. 